\title{
An improved model to estimate trapping parameters in polymeric materials and its application on normal and aged LDPEs
}

\author{
Ning Liu, ${ }^{1,}$ a) Miao He, ${ }^{1}$ Hisham Alghamdi, ${ }^{1}$ George Chen, ${ }^{1}$ Mingli Fu, ${ }^{2}$ Ruihai $\mathrm{Li}^{2}{ }^{2}$ and Shuai $\mathrm{Hou}^{2}$ \\ 1) School of Electronic and Computer Science, University of Southampton, United Kingdom \\ ${ }^{2)}$ Electric Power Research Institute of China Southern Power Grid, Guangzhou, China
}

(Dated: 30 August 2015)

Trapping parameters can be considered as one of the important attributes to describe polymeric materials. In the present paper, a more accurate charge dynamics model has been developed, which takes account of charge dynamics in both volts-on and off stage into simulation. By fitting with measured charge data with the highest $\mathrm{R}$-square value, trapping parameters together with injection barrier of both normal and aged low-density polyethylene (LDPE) samples were estimated using the improved model. The results show that, after long-term ageing process, the injection barriers of both electrons and holes is lowered, overall trap depth is shallower and trap density becomes much greater. Additionally, the changes in parameters for electrons are more sensitive than those of holes after ageing.

Keywords: Space charge model, ageing, trapping parameters, LDPE.

\section{INTRODUCTION}

Space charge accumulation in the insulating materials could lead to local electric field enhancement, which accelerates degradation and ageing of the material. In the last three decades, many investigations have been made to figure out the relationship between space charge and ageing ${ }^{1-4}$. Ageing can be treated as chemical kinetics between unaged state to aged state ${ }^{5}$. The forward transition from unaged state to aged state indicates the process of chemical bonds broken, reorganized and reformed into another configuration in insulation materials while the backward transition refers to the reverse reaction from broken bonds rebounding to original state ${ }^{1,2,5,6}$. Based on physical background that the electrostatic and electromechanical energy storage due to the presence of space charges could accelerate the forward transition rate of ageing and shorten the lifetime, Dissado et al proposed a quantitative model to describe insulation life under the effect of space charges $^{1,2}$. Furthermore, during polarity reversals, insulation performance of the material deteriorated due to space charge accumulation in the insulation materials ${ }^{7}$. Cavallini et al established an empirical model which quantitatively correlates insulation lifetime under polarity reversals and space charges ${ }^{4}$. These models are all supportive to the statement that space charges could be both effects and causes of ageing.

Space charge measurement techniques are generally classified as destructive and non-destructive. Charge detection method in early years was applied as destructive approaches, e.g. Dust-Figure $\operatorname{method}^{8}$. The general principle of these techniques is to section samples into pieces and then detect the charge distribution on the surface of slices with the use of electrostatic

\footnotetext{
a) Electronic mail: nl4g12@soton.ac.uk
}

attraction of powder or field probe ${ }^{9}$. From late 1970s to mid-1990s, many non-destructive methods have been developed to investigate space charge distribution for solid polymeric materials, e.g. laser intensity modulation method $(\text { LIMM })^{10}$, thermal step method $(\text { TSM })^{11}$, pulsed electro-acoustic method $(\mathrm{PEA})^{12}$. In the present paper, PEA technique was employed to measure space charge accumulation under dc voltages.

The storage of space charges in the insulation system could be ascribed to the reason that they can reside within the wide bandgap of insulators without jumping to conduction (valence) band directly. And these localized states, or namely traps, with density $N$, offer charge carriers at an intermediate energy level, i.e. trap depth. Meanwhile, the ability of these traps to capture charge carriers relates to the trapping cross section area $S$. Assuming traps are Coulombic-attractive type and one trap site could only accommodate one charge carrier, thus the cross-section area can be calculated as $S=$ $\pi r^{2}$, where $r$ is the distance between the capturing site and its trapped charge. To summarize, trap density $N$, trap depth $E_{t}$, and trapping cross-sectional area $S$ are generally called trapping parameters, which depict the attributes of traps. In recent years, many approaches have been developed on determination or estimation of these trapping parameters for various insulation materials, especially polyethylene ${ }^{13-18}$. Chen proposed a trapping-detrapping model based on two energy levels. And thereafter in ${ }^{14,19}$, by employing the charge detrapping part in the model established by Chen, trapping parameters of low density polyethylene(LDPE) and also gamma-irradiated LDPE were estimated. It has been revealed that physical and chemical modifications brought by irradiation process is reflected on the changes in trapping parameters. In the case of epoxy resin, Dissado et al proposed a model considering charge detrapping process within three steps ${ }^{15}$. Futhermore, with such model, trapping parameters of different-time aged cross-linked polyethylene (XLPE) cable peelings ${ }^{16}$ 
were evaluated. Similarly, changes in trapping parameters were reported existing between XLPE peelings in different conditions. The basic idea of these two approaches to estimate trapping parameters has something in common: (i) Both numerical models are applied to condition of charge relaxation after the removal of the external voltage; (ii) The trapping parameters were obtained by a fitted curve of specific model parameters with experimental data from only the depolarization tests, i.e. data from polarization test were not exploited; (iii) Observed charge decay after switching off power supply was thought to be caused by detrapping process, or in other words, any charges escaped from the trap sites were presumed to flow away instantly. (iv) Both studies tried to separate traps with a range of energy levels into two equivalent levels ${ }^{13}$ or ranges ${ }^{15}$. And it is noteworthy in works based on Chen's model $^{13,14,19}$, the two energy levels, i.e. shallow and deep traps, have been reported relating with physical and chemical defects in polymeric materials respectively.

In the present paper, a new approach has been proposed, which not only inherits some merits of previous two model works but also improves the accuracy in estimating the trapping parameters of insulation materials. In terms of inheritance, trapping parameters are also determined by optimum curve fitting results with experimental data and meanwhile charge decay data are still a meaningful information to the data fitting process. Except for that, following previous works, the present model also classifies the whole traps as shallow and deep traps. More importantly, comparing to previous works, the new model in the present paper mainly improves in two aspects: (i) In addition to charge relaxation after turning off power supply, the preceding space charge accumulation process during voltage-on condition is included within the simulation works; (ii) Observed space charges in the bulk are considered to consist of trapped charges and mobile charges, which refer to those free charges transporting between traps. Based on the improved model, in the present work, trapping parameters of both unaged and aged polyethylene are extracted. Thus, further comparison of obtained parameters could examine the applicability of such approach to be as ageing marker.

\section{THEORETICAL MODEL}

As mentioned, previous modelling works ${ }^{13,15}$ to estimate trapping parameters are confined to space charge dynamics of depolarization measurements. Also, after the removal of the external voltage, observed space charges are assumed to be trapped charges only and any free charges escaped from traps were thought to be flowing away from electrodes instantly. In our improved model, the observed charges are no longer treated as only trapped charges but includes a non-negligible amount of mobile charges as well. Typically, a space charge signal with homocharge injection can be divided as positive and negative charge regions with thicknesses respectively equalling to $d_{h}$ and $d_{e}$, as shown in Fig. 1. Therefore, the mean number density of net charges $n$ in either region will be calculated as:

$$
n_{h, e}=\frac{Q_{h, e}}{d_{h, e} A}
$$

where $Q_{h, e}$ is total charge amount in either charge region and $A$ is the electrode cross-sectional area. Theoretically, according to the electro-neutrality of bulk, total net charges (including image charges on the surfaces) should be zero. However, the density of net charge in either region equal to the sum of trapped charge and mobile charge density, i.e.:

$$
n_{h, e}=n_{t_{h, e}}+n_{m_{h, e}}
$$

where $n_{t}$ and $n_{m}$ represent trapped and mobile charge number density respectively in either positive $\left(n_{t_{h}}\right.$ and $\left.n_{m_{h}}\right)$ or negative charge zone $\left(n_{t_{e}}\right.$ and $\left.n_{m_{e}}\right)$.

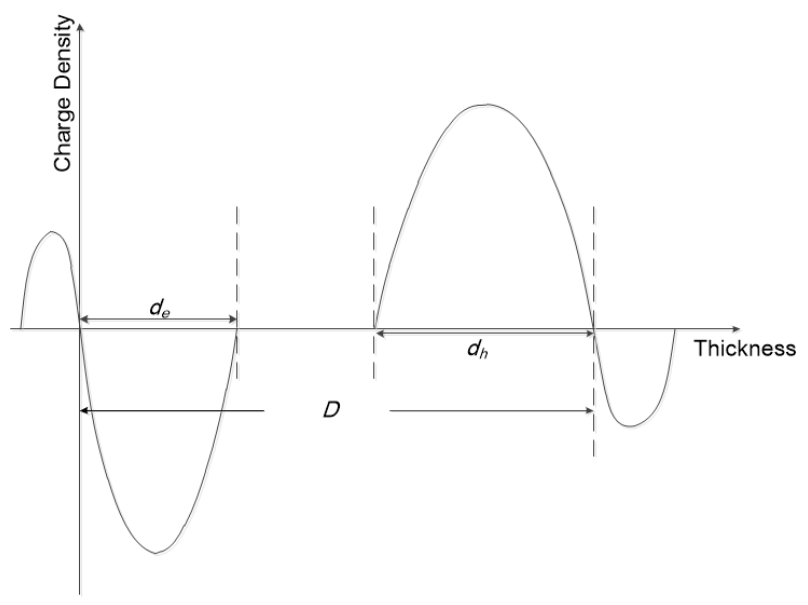

FIG. 1. Space charge profile of homocharge injection denoted with separated charge region of positive and negative.

\section{A. Model Based on Single-level Traps}

To assist in establishing the improved model in which new features are introduced, we start with a single energy level of traps in the material. The concepts are then extended to the two energy levels of trapping/detrapping processes.

\section{Volts-on Condition}

Here, an assumption has been made that the energy depth of all the traps is on the same level. For instance, the changing rate for injected net charge density $n_{h}$ in 
positive charge region under external applied field can be proposed as:

$$
\frac{d n_{h}}{d t}=\frac{J_{h}}{q d_{h}}-P_{h} n_{m_{h}}-\triangle n_{m_{e}}^{\prime}
$$

The first term of Eq. 3 represents the increasing rate of number volumic density of holes coming from the anode by injection. And $J_{h}$ is the injection current density from the anode. With high voltage applied, charge injection behavior at the metal-polyethylene interface was verified conforming to Schottky injection mechanism ${ }^{9,20,21}$, which has already been applied into some previous modelling works on polyethylene ${ }^{18,22,23}$. Such conduction mechanism demonstrates that barrier height at the electrode-dielectric interface is lowered by the applied field and image charge force so that enhanced current density from electrodes takes place. If the interfacial electric field at the anode is $E_{i_{+}}\left(E_{i_{-}}\right.$at the cathode), the injection current density $J_{h}$ coming from the anode can be found as $^{9}$ :

$$
J_{h}=A_{0} T^{2} \exp \left(-\frac{q w_{h}}{k T}\right) \exp \left(\frac{q}{k T} \sqrt{\frac{q E_{i}}{4 \pi \varepsilon_{0} \varepsilon_{r}}}\right)
$$

where $A_{0}$ is a constant term, $k$ is the Boltzmann constant, $T$ is temperature, $\varepsilon_{0}$ is the vacuum permittivity, $\varepsilon_{r}$ is relative permittivity for certain dielectric, and $w_{h}$ stands for the original injection barrier height of holes(or $w_{e}$ for electrons). Normally Schottky constant is written as $\beta_{s c}=\frac{q}{k T} \sqrt{\frac{q}{4 \pi \varepsilon_{0} \varepsilon_{r}}}$. Therefore, Eq. 4 can be simplified as:

$$
J_{h}=A_{0} T^{2} \exp \left(-\frac{q w_{h}}{k T}\right) \exp \left(\beta_{s c} E_{i}^{0.5}\right)
$$

And the second negative term in Eq. 3 describes the decreasing rate of net charge in positive charge layer. Such reduction in the charge layer should be the consequence of the outflow of holes from the local charge region to the opposite electrode. Here, an assumption has been made that there is a fixed proportion $P_{h}\left(\mathrm{~s}^{-1}\right)$ of mobile holes will outflow from the local space charge region, likewise $P_{e}$ for electrons.

The third term $\triangle n_{m_{e}}^{\prime}$ of Eq. 3 represents the increasing rate of the mobile electrons existing in the positive charge region, which are injected from the cathode. To calculate these mobile charges in either space charge region, two situations have to be considered. At every moment, mobile holes of $P_{h} n_{m_{h}}$ shall flow from positive charge region to the other side of bulk meanwhile mobile electrons will flow from negative region towards the positive charge region with amount of $P_{e} n_{m_{e}}$. If $P_{h} n_{m_{h}}>P_{e} n_{m_{e}}$, it can be thought that part of $P_{h} n_{m_{h}}$ will overlap with $P_{e} n_{m_{e}}$ in the flat region between negative layer and positive one, thus showing zero net charge in such region. Residual positive mobile holes of $P_{h} n_{m_{h}}-P_{e} n_{m_{e}}$ will finally go to the negative charge region, i.e. $n_{m_{h}}^{\prime}=P_{h} n_{m_{h}}-P_{e} n_{m_{e}}$ and $\Delta n_{m_{e}}^{\prime}=0$. Likewise, when $P_{h} n_{m_{h}}<P_{e} h n_{m_{e}}$, mobile electrons of
$P_{e} n_{m_{e}}-P_{h} n_{m_{h}}$ will flow to the positive charge layer, i.e. $\triangle n_{m_{e}}^{\prime}=P_{e} n_{m_{e}}-P_{h} n_{m_{h}}$, and $\triangle n_{m_{h}}^{\prime}=0$.

For the changing rate of positive/negative trapped charge density $n_{t}$, it should consists of three terms, i.e.:

$$
\frac{d n_{t_{h}}}{d t}=-R_{e s c}+R_{c a p}-R_{r e c}
$$

where $R_{e s c}$ and $R_{c a p}$ respectively represents the charge escaping rate from the traps and charge capturing rate by traps ${ }^{9}$. And $R_{r e c}$ is the recombination rate of trapped holes/electrons and mobile electrons/holes, which flow from the opposite charge region. Specifically, for positive charge layer, $R_{e s c}, R_{c a p}$ and $R_{r e c}$ can be expressed as:

$$
\begin{gathered}
R_{e s c}=n_{t_{h}} \nu_{0} \exp \left(-\frac{E_{t_{h}}^{\prime}}{k T}\right) \\
R_{c a p}=n_{m_{h}}\left(N_{t_{h}}-n_{t_{h}}\right) S_{h} v_{d_{h}} \\
R_{r e c}=B\left(n_{m_{e}}^{\prime}\right) n_{t_{h}}
\end{gathered}
$$

In Eq. $7, \nu_{0}$ is the escape attempt frequency, approximating as $2 \times 10^{13} \mathrm{~s}^{-1}$ at room temperature ${ }^{9}$. $E_{t_{h}}^{\prime}$ is the modified trap depth based on original trap depth $E_{t_{h}}$ with consideration of Poole-Frenkel lowering $\triangle V_{p f_{h}}$, which will be detailed in section II C, or simply expressed $\mathrm{as}^{9}$ :

$$
E_{t_{h}}^{\prime}=E_{t_{h}}-\triangle V_{p f_{h}}
$$

In Eq. 8 , the rate of charge capture $R_{\text {cap }}$ by traps is proportional to the number density of mobile holes $n_{m_{h}}$, unoccupied trap sites' density $N_{t_{h}}-n_{t_{h}}$, where $N_{h}$ represents the total traps density for holes, and also the drift velocity of charge carriers $v_{d_{h}}$. Physically, such equation can be comprehended as: when mobile charges of number density $n_{m}$ move between trap sites with an averaged drift velocity of $v_{d}$, in travelling time $\Delta t$, the free charges passing through empty traps of number density $N_{t_{h}}-n_{t_{h}}$ with a capture cross section area of $S$ are $n_{m_{h}} v_{d_{h}} \Delta t\left(N_{t_{h}}-n_{t_{h}}\right) S$. If those charges are all captured, the capturing rate can be found as Eq. 8 . With the averaged drift velocity $v_{d}$, the momentum $p$ of the particle moving between two trap sites can be found as:

$$
p=m_{h, e}^{*} v_{d}=q E_{ \pm} t_{d}
$$

where $m_{h, e}^{*}$ is the effective mass of electrons or holes in material, $E_{ \pm}$is local electric field under effect of space charge accumulation in the positive or negative charge region, $t_{d}$ is time of the excited particle moving from one trap to the next. Ignoring Coulombic interactions and assuming the mobile charge is moving straightly along the direction of local electric field (or reverse direction for mobile electrons) between trap sites, therefore $t_{d}$ can be expressed as: $t_{d}=a / v_{d}$, where $a$ is trap separation 
distance. Hence, the average drift velocity of $v_{d}$ can be expressed as:

$$
v_{d}=\sqrt{\frac{q E_{ \pm} a}{m_{h, e}^{*}}}
$$

Eq. 9 gives the recombination rate of trapped positive charges with minor mobile electrons density accumulated in positive layer, i.e. $n_{m_{e}}^{\prime}=\int_{0}^{t} \triangle n_{m_{e}}^{\prime} d t$. This will reduce the trapped charge density in local charge region. Moreover, in Eq. 9, $B$ stands for the recombination rate between positive trapped charges and negative mobile charges.

Substituting the three terms in Eq. 6, a basic differential equation for the trapped charge amount dynamics in positive charge layer can be written as:

$$
\begin{aligned}
\frac{d n_{t_{h}}}{d t}= & -n_{t_{h}} \nu_{0} \exp \left(-\frac{E_{t_{h}}^{\prime}}{k T}\right) \\
& +n_{m_{h}}\left(N_{t_{h}}-n_{t_{h}}\right) S_{h} v_{d_{h}}-B\left(n_{m_{e}}^{\prime}\right) n_{t_{h}}
\end{aligned}
$$

Similarly, the equation for negative trapped charges can be developed.

\section{Volts-off Condition}

After the removal of external voltage, the Schottky injection at the metal-insulator interface could be neglected, because $E_{i_{+,-}}$(electric field at electrodes) included within an exponential term is much lowered. In the depolarization stage, charge carriers should move under field formed by local space charges. For the mobile carriers, the direction of movement should be dependent on the direction of local space charge field. Qualitatively said, mobile positive charges near the anode will escape from the adjacent electrode (anode), whereas those located in the proximity of the interface between negative and positive charge layer will flow to the opposite electrode (cathode). Again, without an extraction barrier at both electrodes, we still postulate a fix portion $P_{h}$ of mobile positive charges will outflow from local charge region. Due to the removal of external voltage, as illustrated in Fig. 2, the charges in either charge layer will travel through bi-directionally dependent on the direction of local space charge field. In other words, only a portion of mobile charge loss will flow towards the opposite electrode. For the sake of simplicity, it was assumed that charges are uniformly distributed in either charge layer and the zero-field locations in negative and positive charge layers are determined as $x_{-}$and $x_{+}$and thickness of the sample is $D$. Hence, mobile electrons/holes existing in positive/negative charge region during volts-off condition can be found as:

i) when $\left(1-\frac{x_{-}}{d_{e}}\right) P_{e} n_{m_{e}}>\left(1-\frac{D-x_{+}}{d_{h}}\right) P_{h} n_{m_{h}}, \triangle n_{m_{e}}^{\prime}=$ $\left(1-\frac{x_{-}}{d_{e}}\right) P_{e} n_{m_{e}}-\left(1-\frac{D-x_{+}}{d_{h}}\right) P_{h} n_{m_{h}}$ and $\triangle n_{m_{h}}^{\prime}=0$.

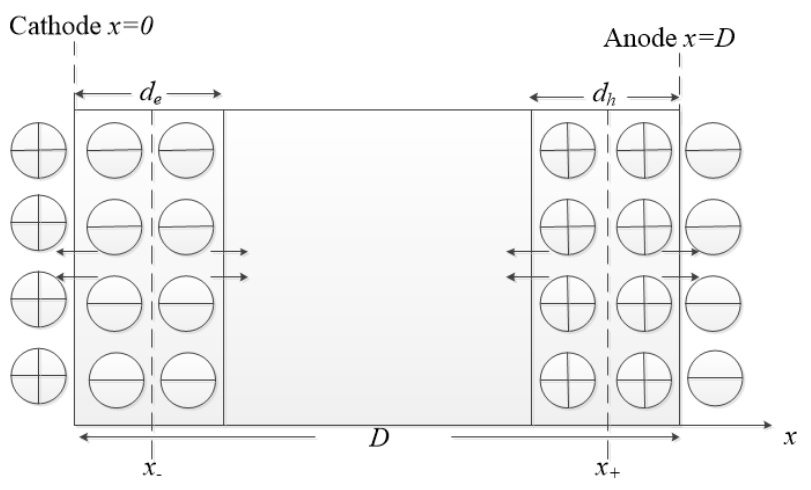

FIG. 2. The charge distribution diagram after removal of external voltage.

ii)when $\left(1-\frac{x_{-}}{d_{e}}\right) P_{e} n_{m_{e}}<\left(1-\frac{D-x_{+}}{d_{h}}\right) P_{h} n_{m_{h}}, \triangle n_{m_{h}}^{\prime}=$ $\left(1-\frac{D-x_{+}}{d_{h}}\right) P_{h} n_{m_{h}}-\left(1-\frac{x_{-}}{d_{e}}\right) P_{e} n_{m_{e}}$ and $\triangle n_{m_{e}}^{\prime}=0$.

The determination of the location $x_{-}$and $x_{+}$will be explained in section II D. Hence, after removal of external voltage, the changing rate of net charge in positive region becomes:

$$
\frac{d n_{h}}{d t}=-P_{h} n_{m_{h}}-\triangle n_{m_{e}}^{\prime}
$$

In terms of the dynamic equation trapped charge during depolarization stage, Eq. 13 will be suitable as well. But the calculation of minority mobile electrons term $\triangle n_{m_{e}}^{\prime}$ should be consistent with that has been calculated for Eq. 14, considering bi-directional flow of mobile charges. Moreover, values of field-dependent parameters need to be modified. These include Poole-Frenkel lowering $\triangle V_{p f_{h}}$, and trapping cross-sectional $S_{h}$. Details will be shown in section II C.

\section{B. Model Based on Dual-level Traps}

In the improved single-level model, all traps in the polymeric material were presumed to be on the same energy level. Factually, these traps might exist with a range of levels in wide band-gap of insulation material. Typically, it was suggested that shallow and deep traps can be correlated with physical and chemical defects in the polymeric material respectively ${ }^{7,14,19,24}$. Physical defects can be created by changes in crystallinity, morphological structure and molecular weight while chemical defects can be produced by oxidation and hydrolysis process ${ }^{9,24}$. Hence, to assign the trapping parameters with more practical meaning, we extend the model from single level to dual energy levels.

With the model of dual levels, Eq. 3 becomes:

$$
\begin{aligned}
\frac{d n_{h}}{d t} & =\frac{J}{q d}-P n_{m_{h}} \\
& =\frac{J}{q d}-P\left(n-n_{t_{h 1}}-n_{t_{h 2}}\right)
\end{aligned}
$$


where $n_{m_{h}}$ is mobile charge density travelling between traps, and $n_{t_{1}}$, and $n_{t_{2}}$ respectively represent the charges captured at shallow and deep energy level. Based on Eq. 13, the changing rate of shallow trapped charges in the positive charge layer can be expressed as:

$$
\begin{aligned}
\frac{d n_{t_{h 1}}}{d t}= & -n_{t_{h 1}} \nu_{0} \exp \left(-\frac{E_{t_{h 1}}-\Delta V_{p f_{h 1}}}{k T}\right) \\
& +n_{m_{h}}\left(N_{t_{h 1}}-n_{t_{h 1}}\right) S_{h 1} v_{d_{h}}-B\left(n_{m_{e}}^{\prime}\right) n_{t_{h 1}}
\end{aligned}
$$

Likely, for changing rate of deep trapped charges, we have:

$$
\begin{aligned}
\frac{d n_{t_{h 2}}}{d t}= & -n_{t_{h 2}} \nu_{0} \exp \left(-\frac{E_{t_{h 2}}-\Delta V_{p f_{h 2}}}{k T}\right) \\
& +n_{m_{h}}\left(N_{t_{h 2}}-n_{t_{h 2}}\right) S_{h 2} v_{d_{h}}-B\left(n_{m_{e}}^{\prime}\right) n_{t_{h 2}}
\end{aligned}
$$

where, for hole traps at shallow and deep levels respectively, $E_{t_{h 1}}$, and $E_{t_{h 2}}$ the original trap depth, $\triangle V_{p f_{h 1}}$ and $\triangle V_{p f_{h 2}}$ are the barrier height lowering due to the Poole-Frenkel effect, $S_{h 1}$, and $S_{h 2}$ capturing cross section area, $v_{d_{h}}$ stands for drift velocity of holes.

Recalling Eq. 14, after the removal of external voltage, the changing rate of net charge in either charge region for dual-level traps model becomes:

$$
\frac{d n}{d t}=-P_{h} n_{m_{h}}=-P_{h}\left(n_{h}-n_{t_{h 1}}-n_{t_{h 2}}\right)
$$

Again Eqs. 16 and 17 still apply for trapped charges dynamics during volts-off condition, but field-dependent parameters have to be adjusted.

\section{Model Parameters Elaboration}

\section{Three-Dimensional Poole-Frenkel Effect}

Poole-Frenkel effect occurs in the material of wide band-gap (e.g. insulating material) where electrons/holes can reside in. The effect demonstrates the Pool-Frenkel mechanism that the potential barrier height of Coulombic attractive traps is deducted by Coulombic force between these charge trapping centers and charge carriers. Therefore, considering the effect of poling field, the trap depth will be reduced in the field direction by certain value $\triangle V_{p f}$. In Eq. 7 , the Poole-Frenkel effect has been considered and the maximum height of the barrier in the field direction is reduced by $\triangle V_{p f}=2\left(\frac{q^{3} E}{4 \pi \varepsilon_{0} \varepsilon_{r}}\right)^{0.5}$. Let $\beta_{p f}=2 \beta_{s c}$, the original 2-D Poole-Frenkel lowering $V_{p f_{0}}$ can be expressed in form:

$$
\triangle V_{p f 0}=\beta_{p f} E^{0.5}
$$

With Poole-Frenkel coefficient $\beta_{p f}$, the Pool-Frenkel conductivity can be expressed $\operatorname{as}^{9}$ :

$$
\sigma=\sigma_{0} \exp \left(-\frac{E_{t}}{2 k T}\right) \exp \left(\frac{\beta_{p f}}{2 k T} E^{0.5}\right)
$$

However, it was pointed out by several investigators that the Poole-Frenkel effect described by Eq. 20 is not dominant behaviour in bulk at high fields for polyethylene materials ${ }^{20,25,26}$. In accordance with Eq. 20 and then by plotting conductivity against the square root of electric field $\left(\sigma-E^{0.5}\right)$, it will give a much higher relative permittivity value(14.2) for LDPE, than the true one $(\sim 2.2)^{20}$. Ieda et al. proposed a corrected three-dimensional Pool-Frenkel model, which takes consideration of the angle $\theta$ between electric field $E$ and electron (or hole)-trap distance $r$, and also an energy level lowering $\delta$ caused by electron/hole-phonon interaction $^{27}$. Such a model will result in the relative permittivity value of LDPE much closer to the true one ${ }^{25,26}$.

In the forward direction of the electric field, considering Poole-Frenkel mechanism with the angle $\theta$, the maximum height of the energy barrier should occur at $r_{p f}=$ $\left(\frac{q}{4 \pi \varepsilon_{0} \varepsilon_{r} E \cos \theta}\right)^{0.5}$. Also in Ref. 27, Ieda proposed a distance $r_{\delta}$ in the opposite direction of electric field, which will determine the energy barrier increment $\triangle V_{\delta}$ reversely along the field. Here, an important assumption is made as: $r_{p f}=r_{\delta}$. Therefore, we have $\delta=\beta(E \cos \theta)^{0.5} / 2$ (see details in Ref. 27). The Poole-Frenkel lowering of the energy barrier therefore becomes:

$$
\triangle V_{p f}(\theta)=\beta_{p f} \frac{(E \cos \theta)^{0.5}}{2}
$$

For $0<\theta<\pi / 2$, the averaged barrier lowering can be calculated according to mean value theorem of integral:

$$
\begin{aligned}
\triangle V_{p f} & =\int_{0}^{\pi / 2} \beta_{p f} \frac{(E \cos \theta)^{0.5}}{2} /(\pi / 2) d \theta \\
& =\frac{\int_{0}^{\pi / 2} \cos \theta^{0.5} d \theta}{\pi} \beta_{p f} E^{0.5}=0.3814 \beta_{p f} E^{0.5}
\end{aligned}
$$

Considering the increased barrier in reverse direction of field, $\triangle V_{\delta}=\triangle V_{p f}$, the rate of charge escaping from trap sites, i.e. Eq. 7, can be re-expressed as:

$$
\begin{aligned}
R_{e s c} & =n_{t} \nu_{0} \exp \left(-\frac{E_{t}}{k T}\right)\left[\exp \left(\frac{\triangle V_{p f}}{k T}\right)+\exp \left(-\frac{\triangle V_{\delta}}{k T}\right)\right] \\
& =2 n_{t} \nu_{0} \exp \left(-\frac{E_{t}}{k T}\right) \cosh \left(\triangle V_{p f} / k T\right)
\end{aligned}
$$

Furthermore, a modified barrier $E_{t}^{\prime}$ under the both impacts of $\triangle V_{p f}$ and $\triangle V_{\delta}$ can be obtained by logarithmizing on both sides of Eq. 23. Therefore, $E_{t}^{\prime}$ is found as:

$$
E_{t}^{\prime}=E_{t}-k T \ln \left[2 \cosh \left(\frac{\triangle V_{p f}}{k T}\right)\right]
$$

And the equivalent barrier height $\triangle V_{p f}^{\prime}$ lowering should be:

$$
\triangle V_{p f}^{\prime}=k T \ln \left[2 \cosh \left(\frac{\triangle V_{p f}}{k T}\right)\right]
$$




\section{Trapping Cross-sectional Area}

Many publications have pointed out that trapping cross sectional area corresponds to two factors: applied electric field ${ }^{28}$ and trap depth ${ }^{29,30}$. An inverse power relationship between capture cross section area and average electric field has been found ${ }^{28}$. A power fit of experimental data for electric field and capture cross section give exponents $n=-1.5$, and -3 respectively for low and high field regions ${ }^{28}$. The threshold electric field between the two power factors falls around $E_{t h}=1.2 \times$ $10^{8} \mathrm{~V} / \mathrm{m}$ (actually much less than electric field in present space charge measurement, $\left.4 \times 10^{7} \mathrm{~V} / \mathrm{m}\right)$. Therefore, we assume in our model, if trap depth remain unchanged, the capture cross section area $S$ is proportional to $E^{-1.5}$.

Furthermore, there is some evidence ${ }^{29,30}$ showing that in dielectrics, deeper traps should have a smaller cross section area. Physically, it can be explained that smaller capture radius will give rise to a greater Coulombic attractive force upon charge carrier, hence forming a deeper trap, which is harder for charge carrier to escape. Especially in Ref. 29, it was proposed that he binding energy $W$ of a Coulombic attractive trap to charge carrier is inversely proportional to the radius of the trap $r$. The binding energy $W$ directly determines the trap depth $E_{t}$. The larger $W$ becomes, the tighter the charge carrier bounds to the trap, i.e. the trap depth becomes deeper. In the present paper, we assumed that $E_{t}$ is proportional to binding energy $W$. Since trapping cross-sectional area $S=\pi r^{2}$, the $S$ can be obtained to be inversely proportional to $E_{t}^{2}$.

Thus, we introduced a capture cross section area value $S_{0}$ at certain trap depth $E_{t 0}$ under electric field $E_{0}$, where in the present paper, let $E_{t 0}=1 \mathrm{eV}$ and $E_{0}=4 \times 10^{7} \mathrm{~V} / \mathrm{m}$. And $S_{0}$ can be determined by the charge simulation curve fitting with experimental data. With such value $S_{0}$, trapping cross sectional area $S$ at any depth of $E_{t}$ under electric field $E$ (less than $1.2 \times 10^{8} \mathrm{~V} / \mathrm{m}$ ) can be expressed as:

$$
S=S_{0}\left(\frac{E_{t 0}}{E_{t}^{\prime}}\right)^{2}\left(\frac{E}{E_{0}}\right)^{-1.5}
$$

In the present model, trap depth $E_{t}$ in Eq. 26 should be replaced by a lowered level $E_{t}^{\prime}$ considering $3-\mathrm{D}$ Poole-Frenkel effect.

\section{Calculation of Electric Fields}

\section{Electric fields near both electrodes}

During the volts-on period, as both negative and positive charges continues to inject into the bulk of dielectric, boundary electric field $E_{i}$ at both cathode and anode will be modified. With number charge density $n(x)$ with respect to the location at $x$-axis, which starts from the cathode, i.e. at cathode $x=0$. According to Gauss's law, the electric field at position $x_{0}$ can be found as:

$$
E\left(x_{0}\right)=\int_{0}^{x_{0}} \frac{n(x) q}{\varepsilon_{0} \varepsilon_{r}} d x
$$

For the sake of simplicity, we assumed that in positive and negative charge region, accumulated holes and electrons are uniformly distributed with charge density $n_{h}$ and $n_{e}$ respectively (see Eq. 1). With the external field $E_{\text {appl }}$ applied, considering the total image charge amount near the cathode $Q_{-}$, the absolute value of electric field at cathode can be found as:

$$
E_{i-}=\left|\frac{Q_{-}}{A \varepsilon_{0} \varepsilon_{r}}-E_{a p p l}\right|
$$

where $A$ is the electrode area. And the field at the anode, it becomes:

$$
E_{i+}=\left|\frac{Q_{-}}{A \varepsilon_{0} \varepsilon_{r}}+\frac{n_{e}(-q) d_{e}+n_{h} q d_{h}}{\varepsilon_{0} \varepsilon_{r}}-E_{\text {appl }}\right|
$$

Therefore, the interface electric field term $E_{i}$ in Eq. 4 (also Eq. 5) can be replaced according to Eq. 28 (cathode) or Eq. 29 (anode). Nevertheless, the images charges amount near the cathode still remains unsolved. This can be calculated as in Eq. $30^{22}$ :

$$
Q_{-}=\int_{0}^{D} \frac{D-x}{D} \rho(x) A d x
$$

where $\rho(x)$ is the net charge density with respect to $x$, which equals to $n(x) q$ (for positive charges, whereas $n(x)(-q)$ for negative charges). Employing such an equation, $Q_{-}$therefore becomes:

$$
Q_{-}=\left[-n_{e}(-q) d_{e}+\frac{n_{e}(-q) d_{e}^{2}-n_{h} q d_{h}^{2}}{2 D}\right] A
$$

With Eq. 31, the electric fields at both electrodes in Eq. 28 and Eq. 29 can be found.

\section{Average electric field across both charge regions}

To calculate the drift velocity $v_{d}=\mu E$, the average electric field of either charge layer has to be calculated. Recalling Eq. 27, with the application of external voltage, at location $x_{0-}$ in negative charge region, the electric field can be expressed in the form:

$$
E_{-}\left(x_{0-}\right)=\left[\frac{Q_{-}}{A \varepsilon_{0} \varepsilon_{r}}+\frac{n_{e}(-q) x_{0-}}{\varepsilon_{0} \varepsilon_{r}}\right]-E_{a p p l}
$$

With such equation, based on the mean value theorem, the absolute value of the averaged field in negative charge layer can be calculated as:

$$
\begin{aligned}
\bar{E}_{-} & =\left|\frac{\int_{0}^{d_{e}}\left\{\left[\frac{Q_{-}}{A \varepsilon_{0} \varepsilon_{r}}+\frac{n_{e}(-q) x}{\varepsilon_{0} \varepsilon_{r}}\right]-E_{a p p l}\right\} d x}{d_{e}}\right| \\
& =\left|\frac{Q_{-}}{A \varepsilon_{0} \varepsilon_{r}}+\frac{n_{e}(-q) d_{e}}{2 \varepsilon_{0} \varepsilon_{r}}-E_{a p p l}\right|
\end{aligned}
$$


Similarly, in the positive charge region, at $x_{0+}$, the field should be:

$$
\begin{aligned}
E_{+}\left(x_{0+}\right) & =\frac{Q_{-}}{A \varepsilon_{0} \varepsilon_{r}}+\frac{n_{e}(-q) d_{e}}{\varepsilon_{0} \varepsilon_{r}}+ \\
& \frac{n_{h} q\left[x_{0+}-\left(D-d_{h}\right)\right]}{\varepsilon_{0} \varepsilon_{r}}-E_{a p p l}
\end{aligned}
$$

Likewise, using Eq. 34, the average field in positive charge layer from $D-d_{h}$ to $D$ is:

$$
\bar{E}_{+}=\left|\frac{Q_{-}}{A \varepsilon_{0} \varepsilon_{r}}+\frac{n_{e}(-q) d_{e}}{\varepsilon_{0} \varepsilon_{r}}+\frac{n_{h} q d_{h}}{2 \varepsilon_{0} \varepsilon_{r}}-E_{a p p l}\right|
$$

After the removal of external voltage, different from volts-on condition, charges in both layers might not only flow to the other side of electrode but also escape from the region through the adjacent electrode, depending on the local space charge field. However, charges moving towards either direction should contribute to the rate of capturing. Hence, if charges move bi-directionally, the location where zero field occurs in either charge layer ( $x_{-}$and $x_{+}$) has to be found respectively. This can be achieved by zeroing Eq. 32 and Eq. 34 without the external field term $E_{a p p l}$. Therefore, we have:

$$
\begin{gathered}
x_{-}=\frac{Q_{-}}{A n_{e} q} \\
x_{+}=\frac{n_{e}}{n_{h}} d_{e}-\frac{Q_{-}}{A n_{h} q}+D-d_{h}
\end{gathered}
$$

If $x_{-} \leq 0$ (or $x_{+} \geq D$ ), this indicates all mobile charges in the negative charge layer (or positive layer) will flow to the other side of bulk. In such case, the averaged field in negative and positive regions during the depolarization stage can be calculated as:

$$
\begin{gathered}
\bar{E}_{-}^{\prime}=\left|\frac{Q_{-}}{A \varepsilon_{0} \varepsilon_{r}}+\frac{n_{e}(-q) d_{e}}{2 \varepsilon_{0} \varepsilon_{r}}\right| \\
\bar{E}_{+}^{\prime}=\left|\frac{Q_{-}}{A \varepsilon_{0} \varepsilon_{r}}+\frac{n_{e}(-q) d_{e}}{\varepsilon_{0} \varepsilon_{r}}+\frac{n_{h} q d_{h}}{2 \varepsilon_{0} \varepsilon_{r}}\right|
\end{gathered}
$$

If $x_{-}>0$ (or $\left.x_{+}<D\right)$, it implies the charges in the negative charge layer (or positive layer) will flow through both directions. Hence, in the case of the negative charge region, the electric field is positive when $x<x_{-}$but negative when $x_{-}<x<d_{e}$, therefore the average field in such a layer should be calculated as:

$$
\begin{aligned}
\bar{E}_{-}^{\prime} & =\left|\frac{\int_{0}^{x_{-}}\left(\frac{Q_{-}}{A \varepsilon_{0} \varepsilon_{r}}-\frac{n_{e} q x}{\varepsilon_{0} \varepsilon_{r}}\right) d x-\int_{x_{-}}^{d_{e}}\left(\frac{Q_{-}}{A \varepsilon_{0} \varepsilon_{r}}-\frac{n_{e} q x}{\varepsilon_{0} \varepsilon_{r}}\right) d x}{d_{e}}\right| \\
& =\left|\frac{Q_{-}\left(2 x_{-}-d_{e}\right)}{A \varepsilon_{0} \varepsilon_{r} d_{e}}-\frac{n_{e} q\left(2 x_{-}^{2}-d_{e}^{2}\right)}{2 \varepsilon_{0} \varepsilon_{r} d_{e}}\right|
\end{aligned}
$$

Similarly, with the zero-field location $x_{+}$in positive charge region, the average field $\bar{E}_{+}^{\prime}$ becomes:

$$
\begin{aligned}
\bar{E}_{+}^{\prime} & =\mid\left[\frac{Q_{-}}{A \varepsilon_{0} \varepsilon_{r}}-\frac{n_{e} q d_{e}+n_{h} q\left(D-d_{h}\right)}{\varepsilon_{0} \varepsilon_{r}}\right] \frac{2 D-2 x_{+}-d_{h}}{d_{h}} \\
& +\frac{n_{h} q\left(2 D^{2}+d_{h}^{2}-2 D d_{h}-2 x_{+}^{2}\right)}{2 \varepsilon_{0} \varepsilon_{r} d_{h}} \mid
\end{aligned}
$$

\section{EXPERIMENTAL}

To validate the established model in the trapping parameters estimation, space charge measurement of normal LDPE was conducted. The experimental data was further used to fit with the model, thus giving the trapping parameters of normal LDPE. Furthermore, space charge dynamics of aged LDPE were also detected. Trapping parameters of aged samples were compared to the those of normal samples.

\section{A. Sample Preparation}

Normal additive-free LDPE films were used for space charge measurement, and the thickness of samples are $170 \pm 10 \mu \mathrm{m}$. Also, some of the normal LDPE films were aged under electric field $6.67 \times 10^{6} \mathrm{~V} / \mathrm{m}$ at $60{ }^{\circ} \mathrm{C}$ for 75 days.

\section{B. Experimental Protocol}

The pulsed electroacoustic (PEA) technique was used for observing dynamics of charge profiles and measurements were made for 60 minutes after the removal of the applied voltage. For LDPE films with slight different thicknesses, the applied voltage was adjusted so the applied field was fixed at $4 \times 10^{7} \mathrm{~V} / \mathrm{m}$ for all the samples. For both normal and aged LDPE samples, three or four consecutive measurements were made. Hence, charge amounts from each layer were averaged by those measured data.

\section{SPACE CHARGE RESULTS AND CALCULATION}

\section{A. Space Charge Dynamics}

From Fig. 3 to Fig. 5, space charge dynamics of both volts-on and volts-off periods using a normal LDPE sample are displayed. Fig. 3 gives space charge dynamics during the volts-on period. In order to obtain the injected space charge profiles in the bulk, the subtraction method was employed to eliminate the capacitive charges on two electrodes $^{31}$, as in Fig. 4. Bipolar charges injection can be observed. But the electrons injection near the 
cathode is remarkably less than holes injection at the anode. After the removal of external voltage, the charge decay result is shown in Fig. 5, the first profile (0s) of which is from subtracted volts-on data at 360s (Fig. 4).

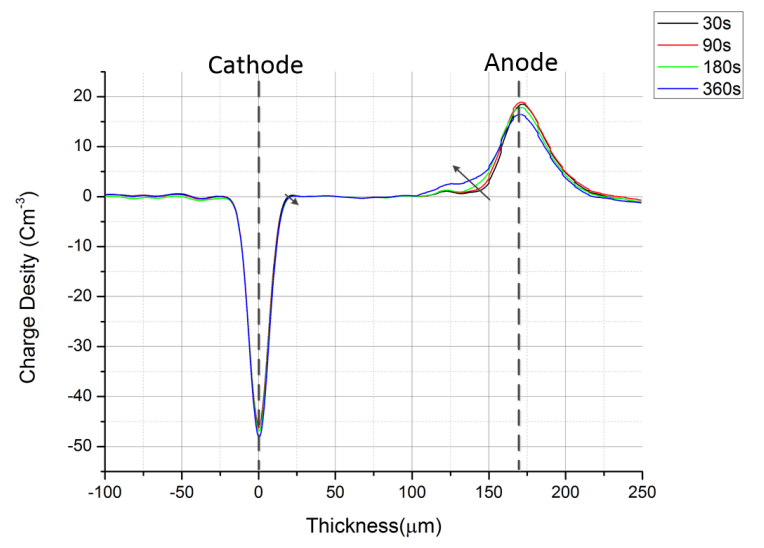

FIG. 3. Space charge dynamics of the $170 \mu \mathrm{m}$ normal LDPE sample under $4 \times 10^{7} \mathrm{~V} / \mathrm{m}$ within 6 minutes.

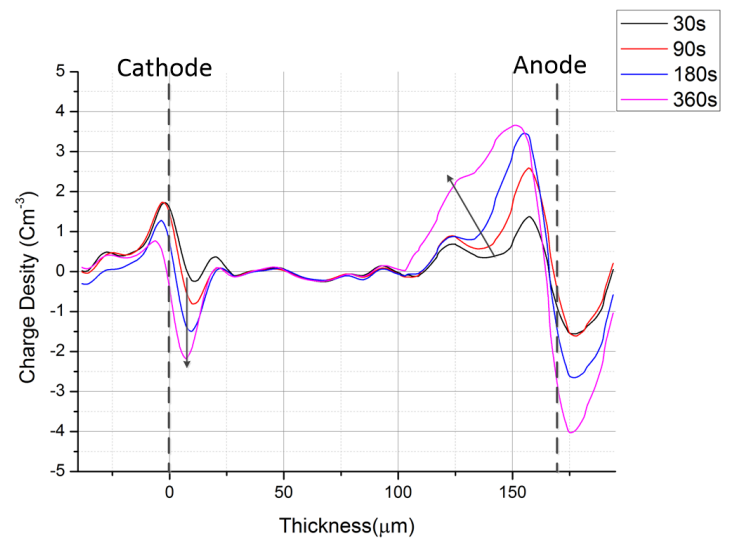

FIG. 4. Space charge dynamics of the $170 \mu \mathrm{m}$ normal LDPE sample under $4 \times 10^{7} \mathrm{~V} /$ mwithin 6 minutes, processed with subtraction algorithm to eliminate capacitive charges.

The space charge dynamics of aged LDPE films were detected, as shown from Fig. 6 to Fig. 8. By comparison with charge data of normal samples, negative injection of aged samples dramatically increases near to the cathode. Moreover, for aged samples, when the power is switched off, negative charges experience a large fall within 60 seconds, as indicated in Fig. 5.

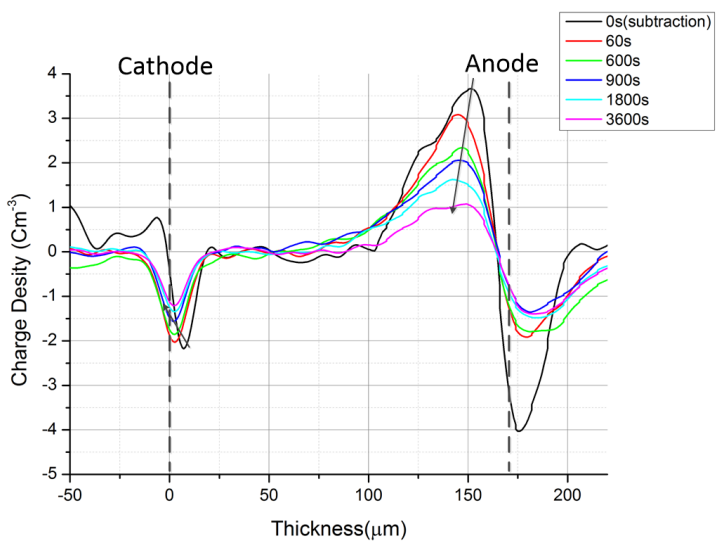

FIG. 5. Space charge dynamics of the $170 \mu \mathrm{m}$ normal LDPE sample during depolarization stage of 3600 s.

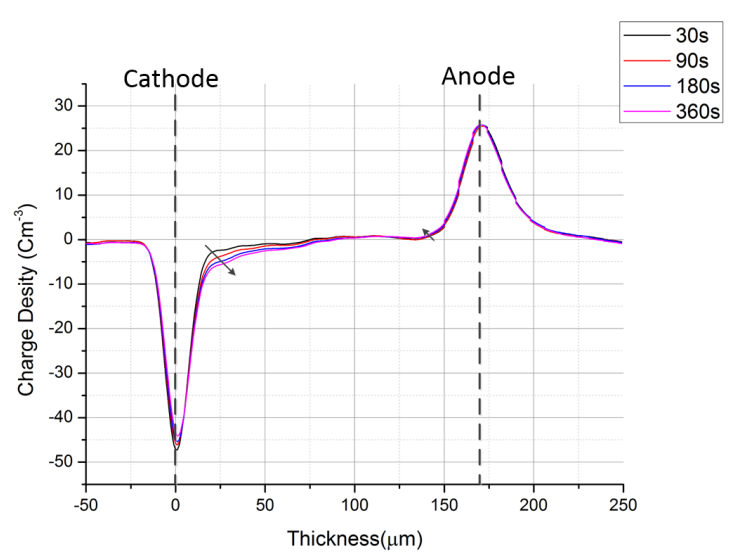

FIG. 6. Space charge dynamics of the $170 \mu \mathrm{m}$ aged LDPE sample under $4 \times 10^{7} \mathrm{~V} / \mathrm{m}$ within 6 minutes.

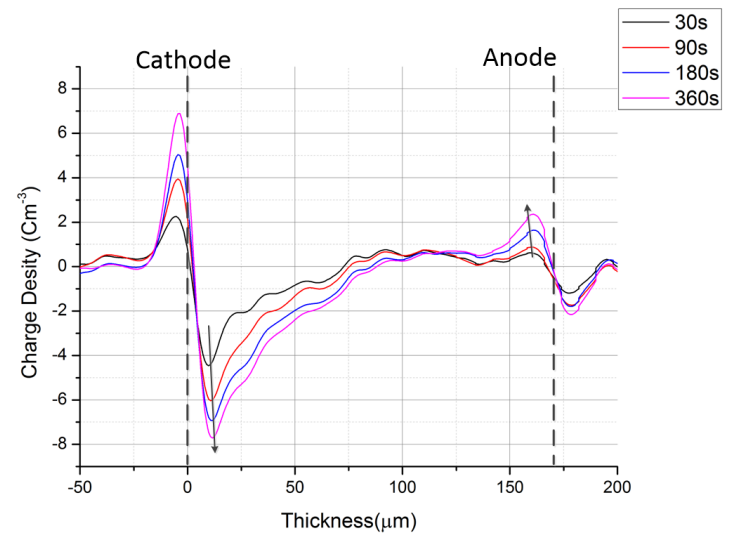

FIG. 7. Space charge dynamics of the $170 \mu \mathrm{m}$ aged LDPE sample under $4 \times 10^{7} \mathrm{~V} / \mathrm{m}$ within 6 minutes, processed with subtraction algorithm to eliminate capacitive charges. 


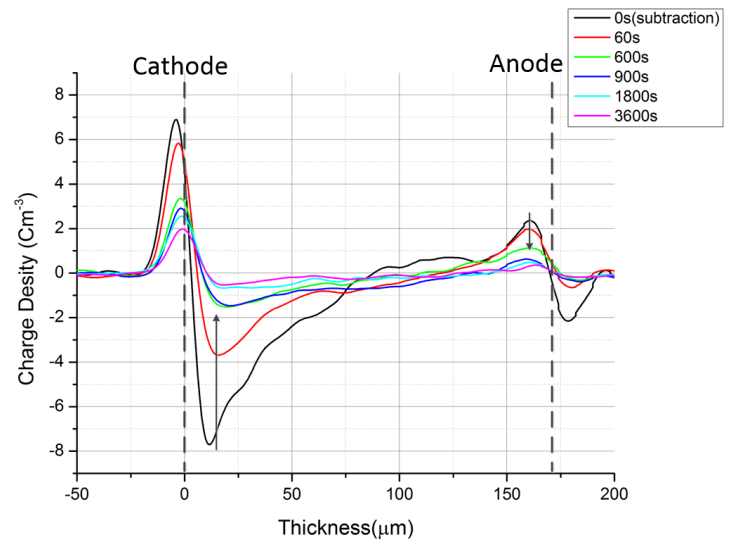

FIG. 8. Space charge dynamics of the $170 \mu \mathrm{m}$ aged LDPE sample during depolarization stage of 3600s.

\section{B. Charge Amount Calculation}

Charge amount in the positive charge layer can be found by the following equation:

$$
Q_{h}=\int_{D-d_{h}}^{D}\left|n_{h}(x, t) q\right| A d x
$$

And in the negative layer, it becomes:

$$
Q_{e}=\int_{0}^{d_{e}}\left|n_{e}(x, t) q\right| A d x
$$

With measured data on several samples, charge amount for both normal and aged LDPE samples can be averaged with error bars shown in Figs. 9 and 10.

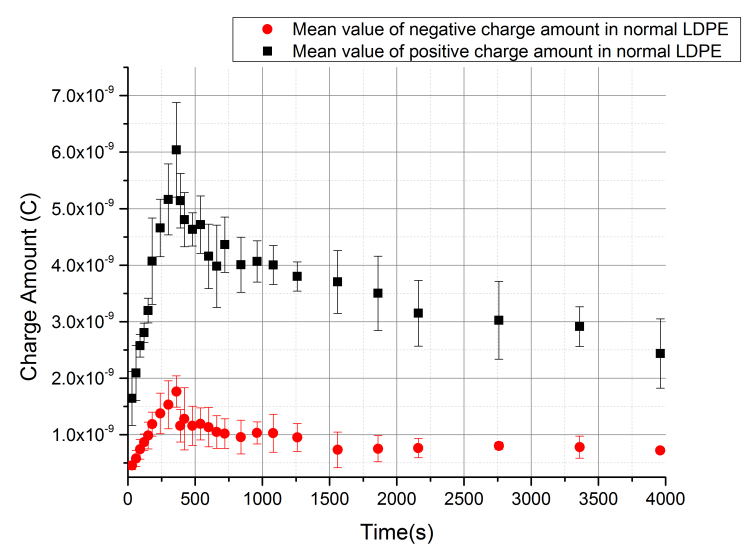

FIG. 9. Averaged positive and negative charge amount in normal LDPE samples.

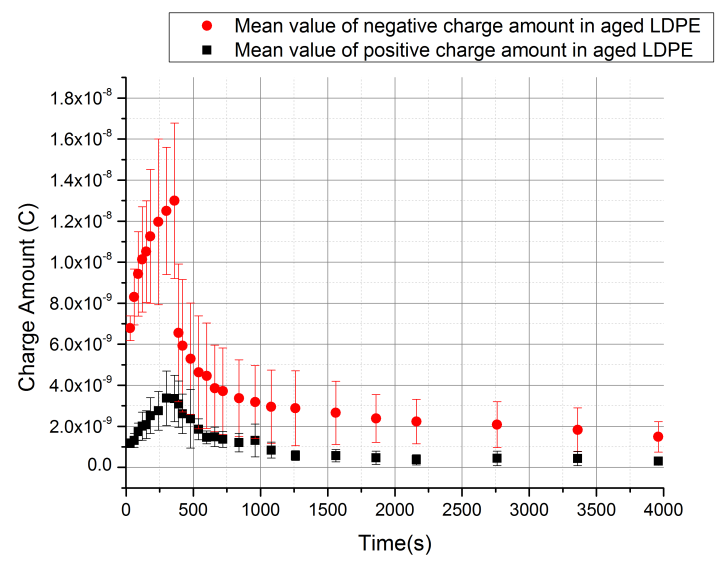

FIG. 10. Averaged positive and negative charge amount in aged LDPE samples.

TABLE I. Values of model constants

\begin{tabular}{cc|cc}
\hline \hline Model Constant & Value & Model Constant & Value \\
\hline$q(\mathrm{C})$ & $1.60 \times 10^{19}$ & $\mathrm{k}\left(\mathrm{JK}^{-1}\right)$ & $1.38 \times 10^{-23}$ \\
\hline$T(\mathrm{~K})$ & 300 & $m_{h, e}^{*}(\mathrm{~kg})$ & $9.11 \times 10^{-31}$ \\
\hline$A_{0}\left(\mathrm{Am}^{-2} \mathrm{~K}^{-2}\right)$ & $1.20 \times 10^{6}$ & $A\left(\mathrm{~m}^{-2}\right)$ & $6.36 \times 10^{-5}$ \\
\hline$B\left(\mathrm{~m}^{3} \mathrm{~s}^{-1}\right)^{\mathrm{a}}$ & $6.40 \times 10^{-19}$ & $\nu_{0}\left(\mathrm{~s}^{-1}\right)^{\mathrm{b}}$ & $2.00 \times 10^{13}$ \\
\hline$\varepsilon_{0}\left(\mathrm{Fm}^{-1}\right)$ & $8.85 \times 10^{-12}$ & $\varepsilon_{r}$ & 2.3 \\
\hline \multirow{2}{*}{$d_{h}(\mu \mathrm{m})^{\mathrm{c}}$} & 55 & $d_{e}(\mu \mathrm{m})^{\mathrm{c}}$ & 20 \\
\hline \multirow{2}{*}{$D(\mu \mathrm{m})^{\mathrm{c}}$} & 40 & & \\
\hline \hline
\end{tabular}

a Values from Ref. 18 and 23.

b Values from Ref. 9 and 20.

c Averaged from measured space charge profiles, where the upper value is for the normal LDPE and the lower one is for the aged LDPE.

\section{PARAMETERS ESTIMATION USING DUAL-LEVEL MODEL}

\section{A. Model Constants}

In the present model, some parameters can be treated as constants in accordance with measured data or the previous literatures, values of which are shown in Table I.

\section{B. Parameters Estimation}

Since analytic solutions cannot be found for ordinary differential equations (see Eq. 15 to 18), the Euler method has been carried out by postulating many small time steps $\Delta t_{s}=1 \mathrm{~s}$ in the whole experimental time $t=3960 \mathrm{~s}$. Numerical solutions of $n_{h, e}$ and $n_{t_{h, e}}$ is found through MATLAB coding.

In the present paper, for both holes and electrons, typical trapping cross sectional area $S_{0}$ mobile charge 
escaping rate constant $P$, injection barrier $w$ and trapping parameters, which include trap density $N$ and depth $E_{t}$, have been set as unknown model parameters remaining to be estimated in the simulation. Through finding the best curve fitting output between experimental data and numerical solutions, i.e. highest $\mathrm{R}$-square value. The $\mathrm{R}$-square is the square of the correlation between the response values and the predicted response values. With a value closer to 1 , it indicates that a greater proportion of variance is accounted for by the model. The highest R-square values for normal and aged LDPE were respectively found as: 0.9205 and 0.9345 . Thus, unknown parameters can be estimated, as shown in Table II. In addition to the table, a typical trapping cross-sectional area $S_{0}$ is found to be $1.00 \times 10^{-30} \mathrm{~m}^{2}$ for both electrons and holes.

Figs. 11 and 12 display the fitting curves obtained through the simulation process by using the improved model of two energy levels for normal and aged LDPE respectively. From those figures, it can be observed that during first 360s under high voltage application, mobiles charges dominates charges in the bulk. Afterwards, when the external voltage is removed at 360s, an interesting feature can be seen that trapped charges continue to increase for about first 120s of depolarization time meanwhile the mobile charges rapidly decrease. When mobile charges reduce to a very low level, the shallow trapped charges start to fall with a relatively slow slope whereas deep trapped charges almost remain flat. This can be ascribed to the trapping cross section area enlargement after the removal of external voltage, i.e. under a much weaker field, according to Eq. 26. Thereafter, a number of mobile charges get retrapped into empty sites. However, as the rapid decrease of mobile charges to nearly zero, little charges can be caught into trapped sites and detrapping process predominates in the bulk. As deep trapped charges are captured at deeper locations in the band gap, it requires a considerably longer time for these charges to escape their localized states.

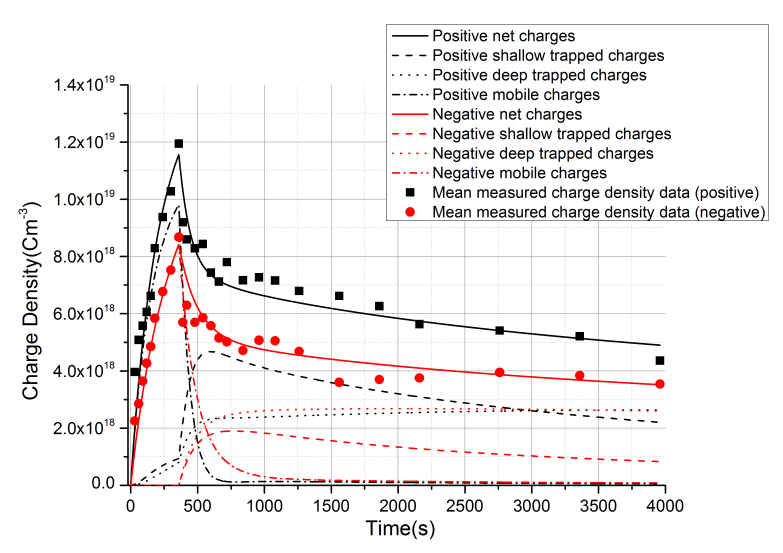

FIG. 11. Simulated curves fitting with experimental data of normal LDPE, based on dual-level model.

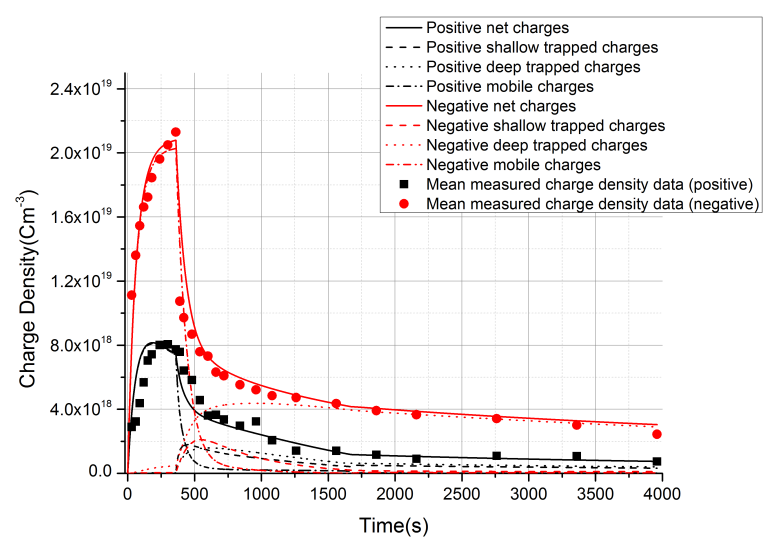

FIG. 12. Simulated curves fitting with experimental data of aged LDPE, based on dual-level model.

\section{ANALYSIS}

When comparing simulated curves with experimental data in Figs. 11 and 12, the major difference is found to be the negative charges' behaviour. After ageing, the negative charges' density increase to a quite high level around $2.4 \times 10^{19}$ and falls to $1.0 \times 10^{19}$ within 60 s after the external power goes off. This indicates, after ageing, much more mobile charges can be injected into the sample with voltage applied, which will immediately run away from the negative charge region after the removal of external voltage, see Fig. 12.

Comparing estimated model parameters of normal LDPE samples to the aged ones (see Table II), the changes can be summarized as:

- In Table II, it can be found that for normal LDPE, injection barrier of holes are lower than that of electrons. After ageing, injection barrier of electrons falls from $1.224 \mathrm{eV}$ to $1.143 \mathrm{eV}$ whereas that of holes experience a little decrease (from $1.185 \mathrm{eV}$ to $1.172 \mathrm{eV})$.

- From Table II, it can be observed that, for holes and electrons, both shallow and deep energy levels of traps are lowered after ageing.

- As displayed in Table II, after ageing, the density of hole traps at both levels increases by a small amount whereas the density of electron traps experiences a much larger increase.

From those model parameters changes after ageing, several causes and consequences can be claimed as:

- The reduction in both holes' and electrons' injection barrier should be explained by deterioration at the surface of the polyethylene material brought by the long-term ageing process. Such modification at metal-insulator interface will 
TABLE II. Estimated parameters of both normal and aged LDPE respectively for holes and electrons, using dual-level modelling.

\begin{tabular}{ccccc}
\hline \hline & \multicolumn{2}{c}{ Normal LDPE } & Aged LDPE \\
\hline Parameters & Holes & Electrons & Holes & Electrons \\
\hline$P\left(\mathrm{~s}^{-1}\right)$ & 0.004 & 0.003 & 0.010 & 0.008 \\
\hline$w(\mathrm{eV})$ & 1.185 & 1.224 & 1.172 & 1.143 \\
\hline$N_{t 1}\left(\mathrm{~m}^{-3}\right)^{\mathrm{a}}$ & $1.38 \times 10^{21}$ & $3.82 \times 10^{18}$ & $1.44 \times 10^{21}$ & $1.38 \times 10^{20}$ \\
\hline$N_{t 2}\left(\mathrm{~m}^{-3}\right)^{\mathrm{b}}$ & $5.43 \times 10^{20}$ & $4.72 \times 10^{18}$ & $5.6 \times 10^{20}$ & 0.967 \\
\hline$E_{t 1}(\mathrm{eV})^{\mathrm{a}}$ & 1.029 & 1.004 & 0.921 & 0.952 \\
\hline$E_{t 2}(\mathrm{eV})^{\mathrm{b}}$ & 1.073 & 1.048 & 1.032 \\
\hline \hline
\end{tabular}

a subscript "1" for shallow traps

b subscript "2" for deep traps

give rise to a much greater charge injection in the aged LDPE samples, especially for electrons.

- In the present paper, long-term combined electrical-thermal accelerated ageing test could bring more physical and chemical defects to the polymeric material. In light of experimental works from Ref. 24, such creation of physical and chemical defects will improve trapping ability of the material, which can be achieved by increasing the trap density and lowering trap depth (see Eq. 26, with shallower trap depth, capturing cross-sectional area expands). Hence, the lowering in trap depth and the increment of trap density after ageing can be elucidated.

\section{CONCLUSION}

In the present paper, an improved charge trapping/detrapping model has been developed which utilizes charge dynamics from both voltage on and off periods. This gives a more accurate account of charge trapping and detrapping processes with the presence of mobile charge transporting between trap sites.

Employing such a model, the behaviour of mobile and trapped charges during volts-on and volts-off period was simulated and injection barrier and trapping parameters of normal and aged LDPE samples can be found for both holes and electrons. Through the comparison of parameters for both types of LDPE sample, it can be found that for both electrons and holes, the injection barrier is lowered, overall trap depth gets shallower and both holes and electrons' trap density increase. The lowering of the injection barrier can be explained by surface modification during the degradation process. The shallower trap depth and the greater trap density are considered to be the consequences of physical and chemical defects brought by the combined thermal-electrical ageing process, which lead to enhanced trapping ability after ageing. Specifically, the injection barrier and trap density of electrons have a much larger variation than those of holes after ageing. This may indicate that parameters of electrons can be used as a diagnostic tool to monitor ageing with better sensitivity.

\section{ACKNOWLEDGEMENT}

The authors (NL\&GC) are grateful for the support received from Electric Power Research Institute of China Southern Power Grid, Guangzhou, China.

${ }^{1}$ L. Dissado, G. Mazzanti, and G. Montanari, "The Incorporation of Space Charge Degradation in the Life Model for Electrical Insulating Materials," IEEE Transactions on Dielectrics and Electrical Insulation 2, 1147-1158 (1995).

${ }^{2}$ L. Dissado, G. Mazzanti, and G. Montanari, "The role of trapped space charges in the electrical aging of insulating materials," IEEE Transactions on Dielectrics and Electrical Insulation 4, 496-506 (1997).

${ }^{3}$ J. Crine, "A Molecular Model to Evaluate the Impact of Aging on Space Charges in Polymer Dielectrics," IEEE Transactions on Dielectrics and Electrical Insulation Vol. 4 No., 487-495 (1997).

${ }^{4}$ A. Cavallini, D. Fabiani, G. Mazzanti, and G. Montanari, "A general model for life estimation of cables under DC stress with voltage-polarity inversions accounting for space-charge effects," in Proceedings of International Symposium on Electrical Insulating Materials (Himeji, 2001) pp. 1-4.

${ }^{5}$ T. Lewis, "Ageing-a perspective," IEEE Electrical Insulation Magazine 17, 6-16 (2001).

${ }^{6}$ T. Lewis, J. Llewellyn, M. Van Der Sluijs, J. Freestone, and R. Hampton, "A new model for electrical ageing and breakdown in dielectrics," in Dielectric Materials, Measurements and Applications, Seventh International Conference on (1996) pp. 220-224.

${ }^{7}$ G. Mazzanti and M. Marzinotto, Extruded cables for high voltage direct current transmission: Advances in reseach and development (John Wiley and Sons, 2013).

${ }^{8}$ N. Ando and F. Numajiri, "Experimental Investigation of Space Charge in XLPE Cable Using Dust Figure," Electrical Insulation, IEEE Transactions on $\mathbf{7 5}$ (1979).

${ }^{9}$ L. Dissado and J. Fothergill, Electrical degradation and breakdown in polymers, 9th ed., edited by P. N. Morgan, D.V. and K. Overshott (Peters Peregrinus Ltd., London, United Kingdom, 1992).

${ }^{10} \mathrm{~S}$. Lang and D. Gas-Gupta, "A technique for determination the polarization distribution in thin polymer electrets using periodic heating," Ferroelectrics 39, 151-154 (2011).

${ }^{11}$ A. Cernomorcenco and P. Notingher, "Application of the thermal step method to space charge measurements in inhomogeneous solid insulating structures: A theoretical approach," Applied Physics Letters 93 (2008).

${ }^{12}$ R. Liu, T. Takada, and N. Takasu, "Pulsed electro-acoustic method for measurement of space charge distribution in power 
cables under both dc and ac electric fields," Journal of Physics D: Applied Physics 26, 986-993 (1993).

${ }^{13} \mathrm{G}$. Chen and $\mathrm{Z}$. Xu, "Charge trapping and detrapping in polymeric materials," Journal of Applied Physics 106, 123707 (2009).

${ }^{14} \mathrm{~T}$. Zhou, G. Chen, R. Liao, and Z. Xu, "Charge trapping and detrapping in polymeric materials: Trapping parameters," Journal of Applied Physics 110, 043724 (2011).

${ }^{15}$ L. Dissado, V. Griseri, W. Peasgood, E. Cooper, K. Fukunaga, and J. Fothergill, "Decay of space charge in a glassy epoxy resin following voltage removal," IEEE Transactions on Dielectrics and Electrical Insulation 13, 903-916 (2006).

${ }^{16}$ A. Tzimas, S. Rowland, and L. Dissado, "The effect of dc poling duration on space charge relaxation in virgin XLPE cable peelings," Journal of Physics D: Applied Physics 43, 2145-2154 (2010).

${ }^{17}$ A. Tzimas, S. Rowland, and L. Dissado, "Effect of electrical and thermal stressing on charge traps in XLPE cable insulation," IEEE Transactions on Dielectrics and Electrical Insulation 19, 2145-2154 (2012).

${ }^{18}$ S. Roy, P. Segur, G. Teyssedre, and C. Laurent, "Description of bipolar charge transport in polyethylene using a fluid model with a constant mobility: model prediction," Journal of Physics D: Applied Physics 37, 298-305 (2004).

${ }^{19} \mathrm{~N}$. Liu and G. Chen, "Changes in charge trapping / detrapping in polymeric materials and its relation with aging," in Electrical Insulation and Dielectric Phenomena, 2012. Annual Report. Conference on (ShenZhen, 2012).

${ }^{20} \mathrm{~J}$. Brunson, Hopping conductivity and charge transport in low density polyethylene, Ph.D. thesis, Utah State University (2010).

${ }^{21} \mathrm{Y}$. Miyoshi and C. K., "Electrical properties of polyethylene single crystals." Japanese Journal of Applied Physics 6, 181-190 (1967).
${ }^{22}$ S. Roy, G. Teyssedre, C. Laurent, G. Montanari, and F. Palmieri, "Description of charge transport in polyethylene using a fluid model with a constant mobility: fitting model and experiments," Journal of Physics D: Applied Physics 39, 1427-1436 (2006).

${ }^{23}$ J. Zhao, Dynamics of space charge and electroluminescence modelling in polyethylene, Ph.D. thesis, University of Southampton (2012).

${ }^{24}$ D. Marsacq, P. Hourquebie, L. Olmedo, and H. Janah, "Effects of physical and chemical defects of polyethylene on space charge behaviour," in Electrical Insulation and Dielectric Phenomena, 1995. Annual Report., Conference on $(123,1995)$ pp. 672-675.

${ }^{25}$ R. Nath, T. Kaura, and M. Perlman, "Steady-state conduction in linear low-density polyethylene with Poole-lowered trap depth," IEEE Transactions on Electrical Insulation 25, 419-425 (1990).

${ }^{26}$ G. Raju, Dielectrics in electric fields (Marcel Dekker, Inc., New York, United States, 2003).

${ }^{27}$ M. Ieda, G. Sawa, and S. Kato, "A Consideration of Poole-Frenkel Effect on Electric Conduction in Insulators," Journal of Applied Physics 42, 3737-3740 (1971).

${ }^{28}$ D. Buchanan, M. Fischetti, and D. DiMaria, "Coulombic and neutral trapping centers in silicon dioxide," Phys. Rev. B 43 (1991).

${ }^{29}$ G. Blaise and W. Sarjeant, "Space charge in dielectrics. energy storage and transfer dynamics from atomistic to macroscopic scale," IEEE Transactions on Dielectrics and Electrical Insulation 5, 779-808 (1998).

${ }^{30}$ V. Afanas, A. Revesz, G. Brown, and H. Hughes, "Deep and Shallow Electron Trapping in the Buried Oxide Layer of SIMOX Structures," J. Electrochem. Soc. 141, 2801-2804 (1994).

${ }^{31}$ N. Liu, C. Zhou, G. Chen, and L. Zhong, "Determination of threshold electric field for charge injection in polymeric materials," Applied Physics Letters 106 (2015). 